\title{
Detection of Alterations in the Gut Microbiota and Intestinal Permeability in Patients With Hashimoto Thyroiditis
}

\section{Leonardo César de Freitas Cayres ${ }^{1 \dagger}$, Larissa Vedovato Vilela de Salis ${ }^{2+}$, Guilherme Siqueira Pardo Rodrigues ${ }^{1}$, André van Helvoort Lengert ${ }^{3}$, Ana Paula Custódio Biondi ${ }^{1}$, Larissa Donadel Barreto Sargentini ${ }^{1}$, João Luiz Brisotti ${ }^{1}$, Eleni Gomes ${ }^{2}$ and Gislane Lelis Vilela de Oliveira ${ }^{1,2,4 *}$}

OPEN ACCESS

Edited by:

Yuan Kun Lee, National University of Singapore, Singapore

Reviewed by:

Huaxi Yi,

Ocean University of China, China Zhihong Sun,

Inner Mongolia Agricultural

University, China

${ }^{*}$ Correspondence:

Gislane Lelis Vilela de Oliveira glelisvilela@gmail.com

tThese authors have contributed equally to this work

Specialty section:

This article was submitted to

Microbial Immunology,

a section of the journal

Frontiers in Immunology

Received: 09 October 2020 Accepted: 04 February 2021 Published: 05 March 2021

Citation:

Cayres LCdF, de Salis LW, Rodrigues GSP, Lengert AvH, Biondi APC, Sargentini LDB,

Brisotti JL, Gomes E and de

Oliveira GLV (2021) Detection of Alterations in the Gut Microbiota and Intestinal Permeability in Patients With Hashimoto Thyroiditis.

Front. Immunol. 12:579140. doi: 10.3389/fimmu.2021.579140
${ }^{1}$ Microbiome Study Group, School of Health Sciences Dr. Paulo Prata, São Paulo, Brazil, ${ }^{2}$ Microbiology Program, Institute of Biosciences, Humanities and Exact Sciences (IBILCE), São Paulo State University (UNESP), São Paulo, Brazil, ${ }^{3}$ Barretos Cancer Hospital, São Paulo, Brazil, ${ }^{4}$ Food Engineering and Technology Department, Institute of Biosciences, Humanities and Exact Sciences, São Paulo State University (UNESP), São José do Rio Preto, Brazil

Hashimoto thyroiditis $(\mathrm{HT})$ is the most common autoimmune disease worldwide, characterized by chronic inflammation and circulating autoantibodies against thyroid peroxidase and thyroglobulin. Patients require hormone replacement with oral levothyroxine, and if untreated, they can develop serious adverse health effects and ultimately death. There is a lot of evidence that the intestinal dysbiosis, bacterial overgrowth, and increased intestinal permeability favor the HT development, and a thyroid-gut axis has been proposed, which seems to impact our entire metabolism. Here, we evaluated alterations in the gut microbiota in Brazilian patients with HT and correlated this data with dietary habits, clinical data, and systemic cytokines and zonulin concentrations. Stool samples from 40 patients with HT and 53 controls were analyzed using real-time PCR, the serum cytokine levels were evaluated by flow cytometry, zonulin concentrations by ELISA, and the dietary habits were recorded by a food frequency questionnaire. We observed a significant increase $(p<0.05)$ in the Bacteroides species and a decrease in Bifidobacterium in samples of patients with HT. In addition, Lactobacillus species were higher in patients without thyroid hormone replacement, compared with those who use oral levothyroxine. Regarding dietary habits, we demonstrated that there are significant differences in the consumption of vegetables, fruits, animal-derived proteins, dairy products, saturated fats, and carbohydrates between patients and control group, and an inverse correlation between animal-derived protein and Bacteroides genus was detected. The microbiota modulation by diet directly influences the inflammatory profile due to the generated microbiota metabolites and their direct or indirect action on immune cells in the gut mucosa. Although there are no differences in systemic cytokines in our patients with HT, we detected increased zonulin concentrations, suggesting a leaky gut in patients with HT. These findings could help understand the development and progression of $\mathrm{HT}$, while further investigations to clarify the underlying mechanisms of the diet-microbiota-immune system axis are still needed.

Keywords: intestinal dysbiosis, inflammatory cytokines, dietary habits, gut microbiota, Hashimoto thyroiditis, autoimmune disease, intestinal permeability 


\section{INTRODUCTION}

The Hashimoto thyroiditis (HT) is an organ-specific autoimmune disease and one of the most common diseases worldwide (1). HT is characterized by chronic inflammation, with tertiary lymphoid follicles development and increased concentrations of circulating autoantibodies against thyroid peroxidase (anti-TPO) and thyroglobulin (anti-Tg) (2). The thyroid parenchyma is replaced by the lymphocytes infiltration, inducing organ enlargement, gland fibrosis, and destruction (3). The progressive thyrocyte depletion leads to decreased thyroid hormone function and clinical hypothyroidism, a condition marked by a reduced metabolic activity in several tissues. The disease is correlated with decreased cardiovascular contractility, coronary artery disease, infertility, dementia, neurosensory and musculoskeletal changes, and reduced colonic activity and orocecal time transit $(4,5)$. Patients require hormone replacement with oral levothyroxine, and if untreated, they can develop serious adverse health effects and ultimately death $(6,7)$.

Hashimoto thyroiditis has become a global public health concern, and the worldwide prevalence reaches $10-12 \%$, affecting 10 times more women, and the peak incidence is between 30 and 50 years old $(5,6)$. In the United States of America, HT affects $\sim 4 \%$ of women aged $18-24$ years and $21 \%$ of women older than 74 years (6). In Europe, the prevalence varies between 0.2 and $5.3 \%$ in the general population, and $7.5 \%$ of women in the United Kingdom presented elevated thyroidstimulating hormone (TSH) concentrations (8). In Brazil, the prevalence of elevated TSH in adult women in Rio de Janeiro was $12.3 \%$, reaching $19.1 \%$ among those over 70 years old. In the metropolitan region of São Paulo, the HT prevalence reached $8.0 \%$, and in an elderly population, the prevalence was 6.5 and $6.1 \%$ for women and men, respectively (9). The economic impact can be significant taking into account the direct effects of the disease, with the HT side effects, which range from depression and to a greater propensity to develop differentiated thyroid cancer and extra-thyroidal cancers (5, 10-13).

Although the HT etiology remains unknown, epidemiological studies suggest that HT is caused by an interaction between genetic and environmental factors (14). The genetic predisposition plays a crucial role in the loss of tolerance to self-antigens and loci linked to immune-modifying genes such as human leukocyte antigens (HLA class I and II) and cytotoxic-T-lymphocyte-associated protein 4 (CTLA-4) could be involved in the autoimmune process. The interactions between these loci and environmental factors might influence disease phenotype and severity (3). The environmental factors that may be involved in the HT triggering include excessive iodine consumption; selenium, iron, zinc, and vitamin D deficiencies; bacterial infections, such as Helicobacter pylori and Yersinia enterocolitica; viral infections, such as hepatitis C, Epstein-Barr virus, and enteroviruses; smoking; pollutants; and chemical agents, and also, factors such as stress, climate, age, and gender (15). More recently, it is hypothesized that gut microbiota might play an important role in triggering HT (16).

There is a lot of evidence that the intestinal dysbiosis, bacterial overgrowth, and increased intestinal permeability (leaky gut) favor HT development, and a thyroid-gut axis has been proposed which seems to impact our entire metabolism $(17,18)$. The commensal gut bacteria influence the innate, cellular, and humoral immunity by interacting with epithelial cells and mucosal immune cells via pattern recognition receptors. Depending on the generated metabolites, microbiota can activate a proinflammatory or anti-inflammatory program (19). Moreover, gut microbiota can affect the thyroid hormone concentrations by controlling iodine uptake, degradation, and enterohepatic recycling of these hormones, and the levothyroxine bioavailability (L-thyroxine). There is a prominent effect of minerals on microbiotahost interactions, mainly by zinc, selenium, and iron. In addition, the microbiota may play role in thyroid disorders by influencing neurotransmitters, the hypothalamus-pituitary axis, the dopamine production, and consequently the TSH secretion (17).

The short-chain fatty acids (SCFAs), metabolites from anaerobic microbiota fermentation, function as an energy source for enterocytes and, together with thyroid hormones, mainly T3, induce enterocyte differentiation, and strengthen the epithelial barrier integrity (20). In addition to SCFAs, the microbiota produce secondary bile acids in the colon, which present systemic effects and interfere with TSH levels (21). Secondary bile acids can regulate type 2 iodothyronine deiodinase (D2) in the gut, molecules involved in thyroid metabolism, and the lipopolysaccharides (LPS) derived from Gram-negative bacteria inhibits intestinal D2 and hepatic D1, and decreases the expression of thyroid hormones in the liver (22).

In animal models, the association between the gut microbiota and thyroid functions has been proposed since 1970s. The depleted microbiota in antibiotic-treated rats induced a decrease in thyroid functions, when evaluated by radioactive iodine uptake (23). In the same line, the germ-free mice present an increase in TSH levels when compared with the conventionally raised mice (22).

In humans, few studies have been carried out in order to understand the relationship between intestinal microbiota and HT. Some recent studies have demonstrated the existence of intestinal dysbiosis in patients with HT and showed an increase in the Bacteroides, EscherichiaShigella, and Parasutterella genera and a decrease in the Bifidobacterium, Lactobacillus, Prevotella, and Dialister genera in the gut microbiota in patients with HT (23). Another study showed an increase in the relative abundance of Blautia, Roseburia, Ruminococcus, Romboutsia, Dorea, Fusicatenibacter, and Eubacterium genera and a decrease in Bacteroides, Faecalibacterium, Prevotella, and Lachnoclostridium genera in the gut microbiota in patients with HT (24).

On the basis of this background and the fact that there are no studies evaluating the gut microbiota in Brazilian patients with HT, the aim of this study was to evaluate the presence of some specific bacteria in stool samples of patients with HT and to correlate these data with dietary habits, clinical data, and systemic cytokines and zonulin concentrations. 


\section{MATERIALS AND METHODS}

\section{Patients With HT and Control Subjects}

Patients diagnosed with HT, with increased TSH and decreased free thyroxine levels (FT4), associated or not associated with elevated anti-TPO and anti-Tg antibodies, were enrolled for this study. The Barretos Cancer Hospital Ethics Committee (Process number 1,359/2017) approved this study, and all participants signed the informed consent form.

About 40 patients with HT aged 23-82 years (mean age $\pm \mathrm{SD}=48.9 \pm 13.3$ years) were enrolled. Fifty-three healthy controls aged $18-79$ years (mean age $\pm \mathrm{SD}=45.6 \pm 16.7$ years) were recruited for the study. Exclusion criteria, for both groups, include the use of anti-inflammatories, immunosuppressant drugs, antibiotics, and vaccination in the last 30 days, with gastrointestinal surgeries, inflammatory bowel diseases, and chronic diarrheas.

After the informed consent, patients and controls answered a food frequency questionnaire (FFQ), containing questions about frequency and consumption of vegetables, fruits, animal-derived proteins, dairy products, carbohydrates, and saturated fat. After that, the peripheral blood was collected, and stool samples were collected within 3-5 days. Table 1 summarizes demographic data and clinical parameters in patients with HT.

\section{DNA Extraction From Stool Samples and Real-Time PCR}

DNA was obtained from $200 \mathrm{mg}$ of stool samples of patients with HT and controls by using QIAamp Fast DNA Stool Mini Kit (QIAGEN Inc., Hilden, Germany), according to the instructions of the manufacturer. The composition of the gut microbiota was determined by specific primers for Bacteroides (Bac), Bifidobacterium (Bif), Clostridium coccoides (Ccoc), Clostridium coccoides-Eubacterium rectale (CIEub), Clostridium leptum (Clept), Lactobacillus (Lac), Prevotella (Prev), and Roseburia (Ros). The primers were described previously and designed by using the 16S rRNA gene sequences from the Ribosomal Database Project (25). PCR was performed by using $7.5 \mu \mathrm{l}$ of Power SYBR Green PCR Master Mix (Applied Biosystems, Life Technologies, Carlsbad, CA, USA), $4.0 \mu \mathrm{l}$ of ultrapure water (Uniscience Corporation, Hialeah, FL, USA), $1.0 \mu \mathrm{l}$ of each primer $(2 \mu \mathrm{M})$, and $1.5 \mu \mathrm{l}$ of DNA (5 ng). The normalization was performed by dividing the DNA copy numbers obtained for target primers (Bac, Bif, Ccoc, CIEub, Clept, Lac, Prev, and Ros) and for the copy numbers of universal primer (Univ). The relative abundance was quantified by using cycle threshold $(\mathrm{Ct})$ values and was expressed in relative expression units (REUs) per $200 \mathrm{mg}$ of stool (26).

\section{Cytokine Serum Quantification by Flow Cytometer}

Approximately $8.5 \mathrm{ml}$ of peripheral blood were collected from patients with HT and healthy individuals in gel tube with clot activator (BD Vacutainer SST II Advance, BD Biosciences, Franklin Lakes, NJ, USA). After collection, samples were incubated at room temperature for $1 \mathrm{~h}$ and centrifuged at 1.372 $\times \mathrm{g}$ for $5 \mathrm{~min}$. The supernatant was withdrawn, and the serum was used for cytokine quantification that was performed by cytometric bead array assay (Human Th1/Th2/Th17 Kit, BD Biosciences, San Jose, CA, USA). The levels of interleukin (IL)2, IL-4, IL-6, IL-10, IL-17A, interferon-gamma (IFN- $\gamma$ ), and tumor necrosis factor (TNF) were detected by a flow cytometer (FACSCanto $^{\mathrm{TM}}$ II, BD Biosciences, Franklin Lakes, NJ, USA). Results were analyzed by BDFCAP array ${ }^{\mathrm{TM}}$ software and were expressed in $\mathrm{pg} / \mathrm{ml}$.

\section{Zonulin Serum Quantification by Sandwich-ELISA}

After peripheral blood collection, samples were incubated in gel tube with clot activator for $50 \mathrm{~min}$ and then centrifuged at $1.372 \times \mathrm{g}$ for $5 \mathrm{~min}$. The serum samples were used for zonulin quantification by using the Human Zonulin ELISA Kit (Elabscience, Bethesda, MD, USA). Standards and samples were added to plates precoated with specific antibodies to human zonulin and incubated for $1 \mathrm{~h}$ at $37^{\circ} \mathrm{C}$. Then, a biotinylated detection antibody specific for human zonulin and avidinhorseradish peroxidase conjugate was added and incubated for $30 \mathrm{~min}$. Unbound and free molecules were washed away. The substrate solution was added to each well and incubated for $15 \mathrm{~min}$. When the enzyme-substrate reaction was blocked by stop solution, the color turned to yellow. The optical density was measured in a spectrophotometer at $450 \mathrm{~nm}$. The standard curve was constructed, and the zonulin concentrations were calculated by converting the obtained optical density in $\mathrm{ng} / \mathrm{ml}$.

\section{Statistical Analysis}

The data extracted from the surveys on dietary habits of patients with HT and controls were analyzed by the Pearson chi-square test, the REUs of the gut microbiota were analyzed by the non-parametric Mann-Whitney $U$-test, and the serum concentrations of cytokines and zonulin were performed by unpaired $t$-test with the Welch's correction. Correlations of the gut microbiota, clinical data, cytokines, and zonulin concentrations were performed by the Spearman's rank correlation coefficient. Values of $p<0.05$ were considered statistically significant.

\section{RESULTS}

\section{Increased Bacteroides and Decreased Bifidobacterium in the Gut Microbiota in Patients}

To analyze the composition of the gut microbiota in patients with HT, we investigated some bacterial species in stool samples by real-time PCR. We detected an increase in the REUs of Bacteroides species in the samples derived from patients (median Bac: 2,344 REU/200 mg stool; $p<0.0001$ ) compared with control subjects (median Bac: $221.3 \mathrm{REU} / 200 \mathrm{mg}$ stool) (Figure 1A). A significant decrease in the REUs of Bifidobacterium species was observed in the stool samples isolated from patients with HT (median Bif: $70.86 \mathrm{REU} / 200 \mathrm{mg}$ stool; $p=0.005$ ) when compared with controls (median Bif: $830.6 \mathrm{REU} / 200 \mathrm{mg}$ stool) (Figure 1B). There are no significant differences $(p>$ 
TABLE 1 | Demographic data and clinical parameters from patients with Hashimoto thyroiditis (HT).

\begin{tabular}{|c|c|c|c|c|c|c|c|c|}
\hline Patients & Gender/Age & Ethnicity & $\begin{array}{l}\text { TSH } \\
(\mu \mathrm{UI} / \mathrm{ml})\end{array}$ & $\begin{array}{l}\text { FT4 } \\
\text { (ng/dl) }\end{array}$ & $\begin{array}{l}\text { TPO Ab } \\
\text { (Ul/ml) }\end{array}$ & $\begin{array}{l}\text { Tg Ab } \\
\text { (UI/ml) }\end{array}$ & $\begin{array}{l}\text { Disease duration } \\
\text { (years) }\end{array}$ & Current treatment \\
\hline HT01 & $F / 28$ & Caucasian & ND & ND & ND & ND & 08 y & LT4 \\
\hline HTO2 & $\mathrm{F} / 57$ & Caucasian & 1.61 & 0.84 & 51.27 & ND & $01 \mathrm{y}$ & NR \\
\hline HT03 & $\mathrm{F} / 35$ & Caucasian & ND & ND & ND & ND & $01 \mathrm{y}$ & NR \\
\hline HTO4 & $F / 28$ & Afrodescendent & ND & ND & ND & ND & 05 y & NR \\
\hline HT05 & $\mathrm{F} / 55$ & Caucasian & ND & ND & ND & ND & 03 y & LT4 \\
\hline HT06 & $F / 29$ & Caucasian & 0.01 & 2.6 & 356 & ND & $13 y$ & NR \\
\hline HT07 & $\mathrm{F} / 56$ & Caucasian & 5.17 & 1.23 & ND & ND & 25 y & LT4 \\
\hline НT08 & $F / 26$ & Caucasian & 6.55 & 1.2 & $>600$ & 241.3 & 04 y & LT4 \\
\hline HT09 & $\mathrm{F} / 42$ & Caucasian & 9.86 & ND & 71 & ND & $12 y$ & LT4 \\
\hline HT10 & $\mathrm{F} / 67$ & Caucasian & 12.21 & 1.1 & 463 & ND & $05 \mathrm{y}$ & LT4 \\
\hline HT11 & $\mathrm{F} / 40$ & Caucasian & 17 & 0.92 & 325 & ND & 03 y & LT4 \\
\hline HT12 & $M / 58$ & Caucasian & 7.58 & 1.2 & ND & ND & 05 y & LT4 \\
\hline HT13 & $\mathrm{F} / 50$ & Afrodescendent & 51 & 0.3 & 109 & 111 & 02 y & LT4 \\
\hline HT14 & $F / 53$ & Afrodescendent & 14 & 0.87 & 1,683 & 6,652 & 42 y & LT4 \\
\hline HT15 & $\mathrm{F} / 54$ & Caucasian & 3.41 & 1.21 & ND & ND & 25 y & NR \\
\hline HT16 & $F / 64$ & Caucasian & 1.53 & 1.84 & $>2,000$ & ND & 25 y & LT4 \\
\hline HT17 & $\mathrm{F} / 52$ & Caucasian & 9.85 & 0.57 & 250 & 11.58 & 05 y & LT4 \\
\hline HT18 & $F / 45$ & Caucasian & 5.51 & 0.62 & 160 & 137.8 & $02 \mathrm{y}$ & LT4 \\
\hline HT19 & $F / 63$ & Afrodescendent & ND & ND & ND & ND & $17 \mathrm{y}$ & LT4 \\
\hline HT20 & $F / 53$ & Caucasian & ND & ND & ND & ND & $10 y$ & LT4 \\
\hline HT21 & $\mathrm{F} / 82$ & Afrodescendent & ND & ND & ND & ND & $10 y$ & NR \\
\hline HT22 & $F / 54$ & Caucasian & ND & ND & 211.8 & 56.53 & $20 y$ & LT4 \\
\hline HT23 & $F / 38$ & Afrodescendent & ND & ND & ND & ND & $14 y$ & LT4 \\
\hline HT24 & F/65 & Caucasian & 4.52 & ND & ND & ND & $10 y$ & LT4 \\
\hline HT25 & $\mathrm{F} / 52$ & Afrodescendent & 10.1 & ND & ND & ND & 06 y & LT4 \\
\hline HT26 & $F / 65$ & Afrodescendent & 4.72 & ND & ND & ND & 05 y & LT4 \\
\hline HT27 & M/62 & Caucasian & 16.8 & 2.05 & 10.82 & 42.81 & 03 y & LT4 \\
\hline HT28 & $\mathrm{F} / 47$ & Caucasian & 1.26 & 1.1 & ND & ND & $21 \mathrm{y}$ & LT4 \\
\hline HT29 & $F / 35$ & Caucasian & 9.48 & ND & ND & ND & 07 y & LT4 \\
\hline НТ30 & $F / 51$ & Afrodescendent & 3.2 & 0.89 & 270 & 143.23 & $18 y$ & NR \\
\hline HT31 & $\mathrm{F} / 45$ & Caucasian & 4.33 & 1.06 & 159 & 214 & $04 y$ & LT4 \\
\hline HT32 & M/66 & Caucasian & ND & ND & ND & ND & 03 y & NR \\
\hline HT33 & $M / 56$ & Caucasian & 0.52 & 1.73 & 970 & 26 & $0.9 y$ & LT4 \\
\hline НТ34 & $F / 23$ & Caucasian & 2.03 & 0.96 & 1,840 & 8.58 & 05 y & LT4 \\
\hline HT35 & $F / 44$ & Caucasian & 0.97 & ND & ND & ND & $07 \mathrm{y}$ & LT4 \\
\hline НТ36 & $F / 45$ & Caucasian & ND & ND & ND & ND & ND & LT4 \\
\hline HT37 & $F / 49$ & Caucasian & 4.91 & 0.95 & 0.52 & 57 & 07 y & LT4 \\
\hline HT38 & $F / 43$ & Caucasian & 0.48 & 1.1 & 366 & 5.06 & 02 y & LT4 \\
\hline НТ39 & $F / 52$ & Caucasian & 4.89 & 1.24 & 282 & $>1,000$ & $10 y$ & LT4 \\
\hline HT40 & $\mathrm{F} / 27$ & Caucasian & ND & ND & ND & ND & ND & LT4 \\
\hline
\end{tabular}

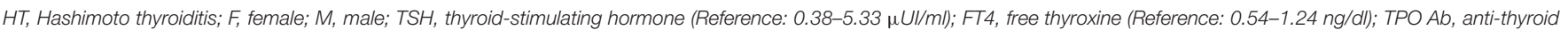

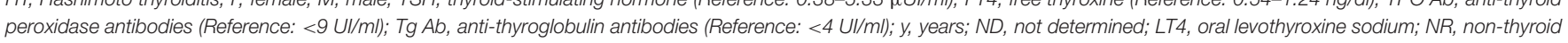
hormone replacement.

0.05) in the REU/200 mg stool obtained from Clostridium coccoides (median Ccoc: 43.51), Clostridium coccoides-Eubacteria rectale (median CIEub: 48.03), Clostridium leptum (median Clept: 1.060), Lactobacillus (median Lac: 0.974), Prevotella (median Prev: 12.53), and Roseburia species (median: 385.8 ) in stool samples of patients with HT, when compared with the healthy individuals (median Ccoc: 51.21; CIEub: 78.04; Clept:
1,762; Lac: 4.933; Prev: 0.530; and Ros: 635.9, respectively) (Figures 1C-H).

\section{Dietary Habits and Correlations With the Gut Microbiota}

To evaluate the dietary habits from patients and controls, we applied an FFQ regarding the frequency of consumption 

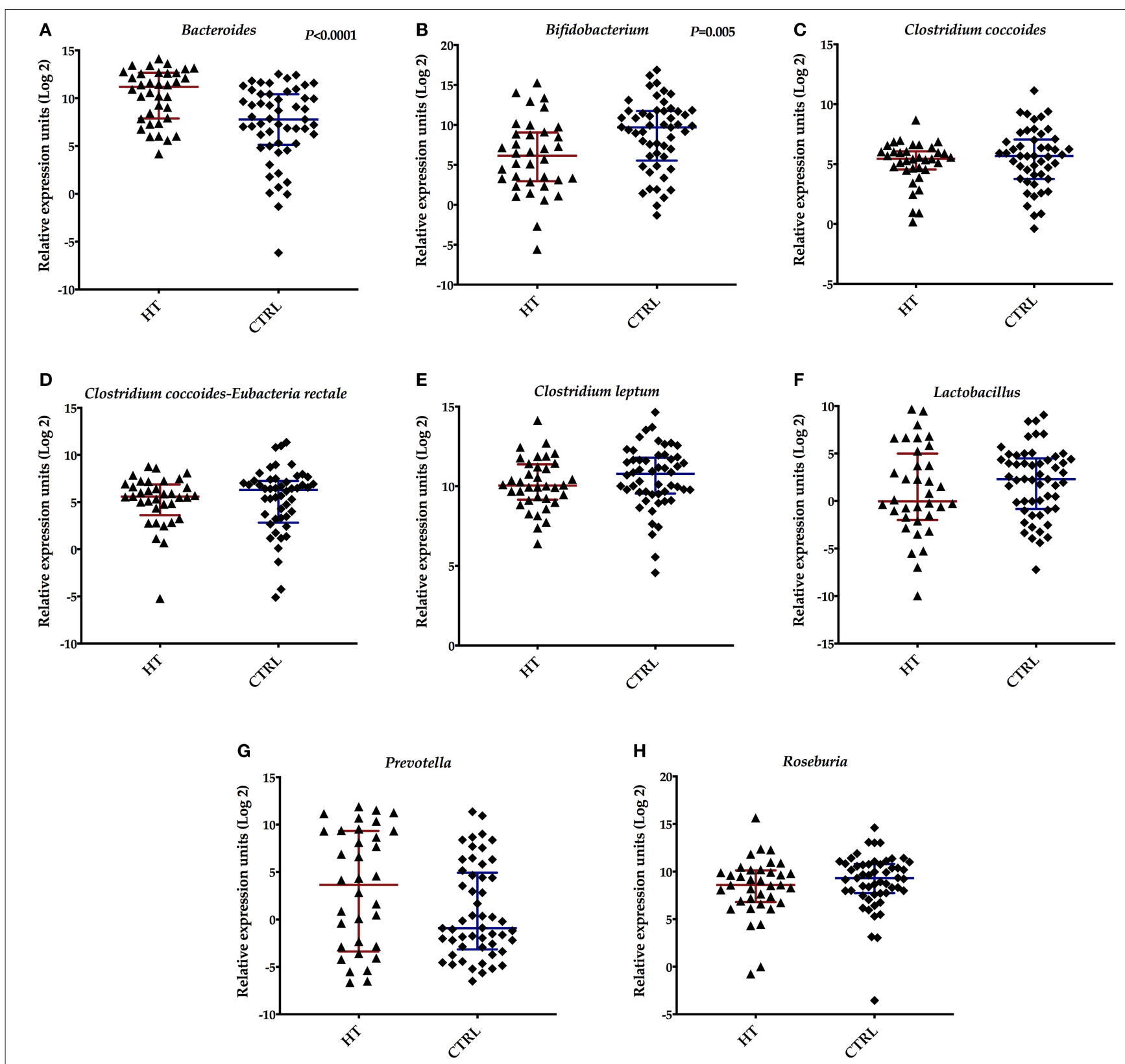

FIGURE 1 | Relative expression units (REU) of the intestinal microbiota found in stool samples from patients with Hashimoto thyroiditis (HT) and healthy controls (CTRL). (A) Bacteroides, (B) Bifidobacterium, (C) Clostridium coccoides, (D) Clostridium coccoides-Eubacterium-rectale, (E) Clostridium leptum, (F) Lactobacillus, (G) Prevotella, and (H) Roseburia species. Bars represent the median with interquartile range of REU per $200 \mathrm{mg}$ of stool.

of vegetables, fruits, animal-derived proteins, dairy products, saturated fats, and carbohydrates. The interviewed subjects reported the daily ingestion of vegetables [patients $(\mathrm{HT})=59 \%$; controls $(\mathrm{C})=53.3 \%$; fruits $(\mathrm{HT}=38.5 \%$; $\mathrm{C}=43.3 \%)$, proteins $(\mathrm{HT}=51.3 \% ; \mathrm{C}=30 \%)$, dairy products $(\mathrm{HT}=48.7 \% ; \mathrm{C}=60 \%)$, saturated fats $(\mathrm{HT}=17.9 \% ; \mathrm{C}=16.7 \%)$, and carbohydrates $(\mathrm{HT}$ $=38.5 \%$; $\mathrm{C}=43.3 \%)$ ]. When we compared the dietary habits between patients with HT and controls, we observed a significant difference $(p<0.05)$ in the consumption of vegetables, fruits, proteins, dairy products, saturated fats, and carbohydrates. The results concerning the dietary habits from patients and controls were shown in Table 2.

In order to detect correlations between diet and gut microbiota composition in patients with $\mathrm{HT}$, we used the consumption frequencies and the REUs of Bacteroides, Bifidobacterium, Clostridium coccoides, Clostridium coccoidesEubacteria rectale, Clostridium leptum, Lactobacillus, Prevotella, and Roseburia. We found an inverse correlation $(p=0.002 ; r=$ -0.61 ) between animal-derived protein consumption by patients and the REUs of Bacteroides species. 
TABLE 2 | Dietary habits of the patients with HT and control subjects.

\begin{tabular}{|c|c|c|c|}
\hline $\begin{array}{l}\text { Dietary variables } \\
\text { (consumption } \\
\text { frequency) }\end{array}$ & $\begin{array}{c}\text { Patients with } \\
\text { HT N (\%) }\end{array}$ & $\begin{array}{c}\text { Controls } N \\
(\%)\end{array}$ & $p$-value \\
\hline \multicolumn{4}{|l|}{ VEGETABLES } \\
\hline $\begin{array}{l}\text { Less than once per } \\
\text { month }\end{array}$ & $1(2.6 \%)$ & 0 & $p<0.001$ \\
\hline 1-3 times per month & $2(5.1 \%)$ & $2(6.7 \%)$ & \\
\hline 1-2 times a week & $2(5.1 \%)$ & $4(13.3 \%)$ & \\
\hline On most days & $11(28.2 \%)$ & 8 (26.7\%) & \\
\hline Every day & 23 (59\%) & 16 (53.3\%) & \\
\hline \multicolumn{4}{|l|}{ FRUITS } \\
\hline $\begin{array}{l}\text { Less than once per } \\
\text { month }\end{array}$ & $3(7.7 \%)$ & 0 & $p<0.001$ \\
\hline 1-3 times per month & 4 (10.3\%) & $4(13.3 \%)$ & \\
\hline 1-2 times a week & 7 (17.9\%) & 8 (26.7\%) & \\
\hline On most days & $10(25.6 \%)$ & $5(16.7 \%)$ & \\
\hline Every day & 15 (38.5\%) & $13(43.3 \%)$ & \\
\hline \multicolumn{4}{|l|}{ PROTEINS } \\
\hline 1-3 times per month & $1(2.6 \%)$ & $1(3.3 \%)$ & $p<0.001$ \\
\hline 1-2 times a week & $6(15.4 \%)$ & $11(36.7 \%)$ & \\
\hline On most days & 12 (30.8\%) & 9 (30.0\%) & \\
\hline Every day & 20 (51.3\%) & $9(30.0 \%)$ & \\
\hline \multicolumn{4}{|l|}{ DAIRY PRODUCTS } \\
\hline Never consumes & $5(12.8 \%)$ & $1(3.3 \%)$ & $p<0.001$ \\
\hline $\begin{array}{l}\text { Less than once per } \\
\text { month }\end{array}$ & $3(7.7 \%)$ & 0 & \\
\hline 1-3 times per month & $4(10.3 \%)$ & $3(10.0 \%)$ & \\
\hline 1-2 times a week & $3(7.7 \%)$ & $6(20.0 \%)$ & \\
\hline On most days & $5(12.8 \%)$ & $2(6.7 \%)$ & \\
\hline Every day & $19(48.7 \%)$ & $18(60.0 \%)$ & \\
\hline \multicolumn{4}{|l|}{ SATURATED FAT } \\
\hline Never consumes & 7 (17.9\%) & $7(23.3 \%)$ & $p=0.005$ \\
\hline $\begin{array}{l}\text { Less than once per } \\
\text { month }\end{array}$ & $2(5.1 \%)$ & 1 (3.3\%) & \\
\hline 1-3 times per month & 8 (20.5\%) & 10 (33.3\%) & \\
\hline 1-2 times a week & $11(28.2 \%)$ & $6(20.0 \%)$ & \\
\hline On most days & $4(10.3 \%)$ & 1 (3.3\%) & \\
\hline Every day & 7 (17.9\%) & $5(16.7 \%)$ & \\
\hline \multicolumn{4}{|l|}{ CARBOHYDRATES } \\
\hline Never consumes & $1(2.6 \%)$ & 0 & $p<0.001$ \\
\hline $\begin{array}{l}\text { Less than once per } \\
\text { month }\end{array}$ & $2(5.1 \%)$ & 0 & \\
\hline $1-3$ times per month & $6(15.4 \%)$ & $2(6.7 \%)$ & \\
\hline 1-2 times a week & $13(33.3 \%)$ & 6 (20.0\%) & \\
\hline On most days & $2(5.1 \%)$ & 9 (30.0\%) & \\
\hline Every day & 15 (38.5\%) & $13(43.3 \%)$ & \\
\hline
\end{tabular}

\section{Correlations Between the Gut Microbiota and Clinical Data}

To evaluate the connection between gut microbiota composition and clinical parameters, we correlated the REUs of Bacteroides, Bifidobacterium, Clostridium coccoides, Clostridium coccoidesEubacteria rectale, Clostridium leptum, Lactobacillus, Prevotella, and Roseburia with concentrations of TSH, FT4, TPO, and Tg autoantibodies. We detected positive correlations among the REUs of Clostridium coccoides $(p=0.023 ; r=0.40)$ and Clostridium coccoides-Eubacteria rectale $(p=0.010 ; r=0.45)$ and in the TSH levels (Figure 2A). The REUs of Roseburia species inversely correlated $(p=0.015 ; r=-0.49)$ with FT4 levels (Figure 2B). We also found a positive correlation between Clostridium coccoides $(p=0.040 ; r=0.30)$ and disease duration (Figure 2C). There are no correlations $(p>0.05)$ among gut microbes and TPO and Tg autoantibodies. Besides that, we observed significant differences in the REUs of Lactobacillus between patients who did thyroid hormone replacement therapy $(N=28)$ and those who did not $(N=8)$ (median Lac LT4: 0.8065 REU/200 mg stool; median Lac NR: 47.73; $p=0.020$; Figure 2D).

\section{Systemic Cytokine Profile in Patients With HT Was Similar to Controls}

To investigate whether the alterations of intestinal microbiota impacted on systemic cytokine profile in patients with HT, we analyzed the serum concentrations of IL-2, IL-4, IL-6, IL-10, IL$17 \mathrm{~A}, \mathrm{IFN}-\gamma$, and TNF by flow cytometer. Significant differences in these cytokines were not detected $(p>0.05)$ (mean \pm SE for IL-2: $0.3369 \pm 0.0638 \mathrm{pg} / \mathrm{ml}$; IL- $4: 0.4081 \pm 0.0542 \mathrm{pg} / \mathrm{ml}$; IL6: $1.121 \pm 0.1599 \mathrm{pg} / \mathrm{ml}$; IL-10: $0.3192 \pm 0.0333 \mathrm{pg} / \mathrm{ml}$; IL-17A: $0.5835 \pm 0.2388 \mathrm{pg} / \mathrm{ml} ; \mathrm{IFN}-\gamma: 0.4473 \pm 0.1023 \mathrm{pg} / \mathrm{ml}$; and TNF: $1.154 \pm 0.1090 \mathrm{pg} / \mathrm{ml}$ ) when compared with controls (mean $\pm \mathrm{SE}$ for IL-2: $0.3800 \pm 0.0527 \mathrm{pg} / \mathrm{ml}$; IL-4: $0.3791 \pm 0.0541 \mathrm{pg} / \mathrm{ml}$; IL6: $1.375 \pm 0.2492 \mathrm{pg} / \mathrm{ml}$; IL-10: $0.2884 \pm 0.0414 \mathrm{pg} / \mathrm{ml}$; IL-17A: $0.3981 \pm 0.1985 \mathrm{pg} / \mathrm{ml}$; IFN- $\gamma: 0.3438 \pm 0.0951 \mathrm{pg} / \mathrm{ml}$; and TNF: $1.317 \pm 0.1711 \mathrm{pg} / \mathrm{ml}$ ) (Figures 3A-G).

\section{Detection of Increased Intestinal Permeability in Patients With HT}

In order to find out whether patients with HT were presented with a leaky gut, since alterations in the gut microbiota were detected, we evaluated the serum zonulin concentrations in patients with $\mathrm{HT}$ and controls. The zonulin serum concentrations were significantly increased $(p=0.002)$ in samples of patients with HT (mean \pm SE: $30.92 \pm 2.36 \mathrm{ng} / \mathrm{ml}$ ) when compared with controls (mean \pm SE: $19.01 \pm 2.98 \mathrm{pg} / \mathrm{ml}$ ) (Figure 4A). Besides that, the zonulin concentrations positively correlated with systemic IFN- $\gamma$ levels $(p=0.042 ; r=0.52$; Figure 4B) and inversely correlated with IL-2 concentrations ( $p$ $=0.042 ; r=-0.51$ ) in patients with HT (Figure 4B).

\section{DISCUSSION}

There is a lot of evidence that alterations in the gut microbiota are associated with autoimmune diseases development $(16,27-$ 29). Researchers suggested that the molecular mimicry, bystander T-cell activation, post-translational modification of luminal proteins by altered microbiota, a shift to proinflammatory milieu in the gut mucosa, intestinal dysbiosis, bacterial overgrowth, and leaky gut could contribute to autoimmunity and HT trigger in genetically susceptible individuals (17, $18,30,31)$. The commensal microbiota play an important 

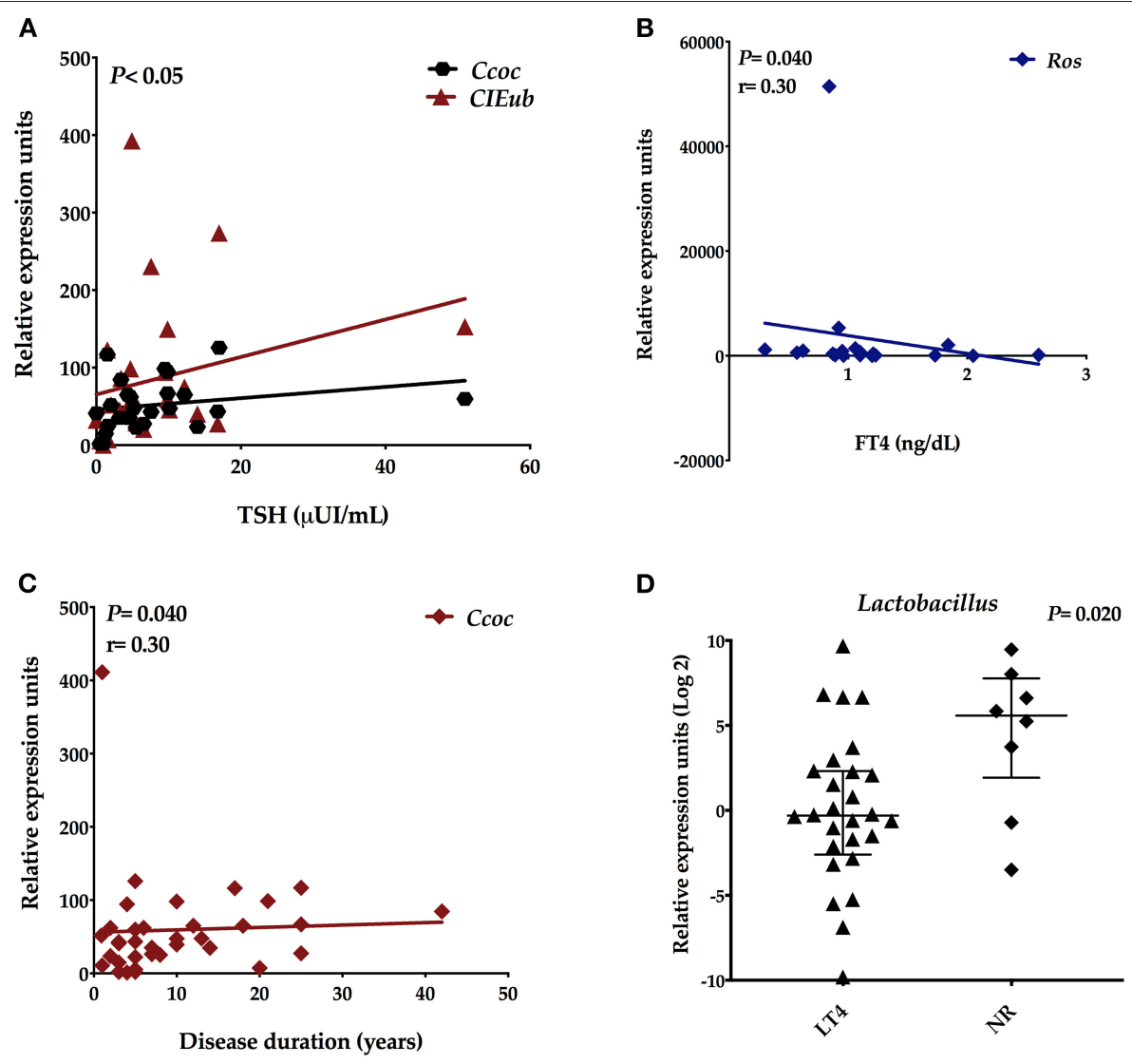

FIGURE 2 | Spearmans' correlation between the relative expression units (REU) of the intestinal microbiota and clinical data. (A) REU of Clostridium coccoides and Clostridium coccoides-Eubacterium-rectale with TSH concentrations, (B) REU of Roseburia with FT4 levels, (C) REU of Clostridium coccoides with disease duration, and (D) REU of Lactobacillus species in treated patients (LT4) and patients with no hormone replacement (NR).

role in our physiological processes, influencing the mucosal innate and adaptive immunity and host metabolism, including carbohydrate digestion and fermentation, vitamin synthesis, hormones production and secretion, secondary bile acid synthesis, absorption of micronutrients, including iron, zinc, and iodine, which directly affect thyroid function $(22,32)$. In the present study, we investigated the alterations in the gut microbiota in Brazilian patients with HT and correlated these data with dietary habits, clinical data, and systemic cytokines and zonulin concentrations.

There are four studies that compared the gut microbiota from patients with euthyroid and hypothyroid HT in Chinese population, and researchers detected intestinal dysbiosis when compared with healthy controls $(23,24,33,34)$. Ishaq et al. (23) investigated the microbial composition in 29 patients with HT and 12 healthy controls by PCR-DGGE, real-time PCR, and $16 \mathrm{~S}$ pyrosequencing $(17,19)$. Authors showed a reduction in Firmicutes and Bacteroidetes, and increased Proteobacteria and Cyanobacteria phyla in HT samples. Findings of the DGGE analysis showed a different fingerprint between patients and controls and the predominance of pathogenic microbes, such as Bacteroides uniformis, B. pyogenes, B. vulgates, Shigella dysenteriae, B. intestinalis, Escherichia coli, Sporomusa ovate, Shigella flexneri, and Bacillus species. By real-time PCR, authors observed a significant decrease in copy numbers of Bifidobacterium and Lactobacillus, and an increase in E. coli in patients with HT. By the $16 \mathrm{~S}$ pyrosequencing, researchers detected a predominance of Bacteroides, Escherichia-Shigella, and Parasutterella genera, and a reduction in Prevotella and Dialister genera in patients with HT (23).

Another study examined the intestinal microbiota in 28 patients with euthyroid and 16 controls by $16 \mathrm{~S}$ sequencing and showed that Firmicutes/Bacteroidetes ratio was increased and the Lachnospiraceae family was prevalent in stool of patients with HT. The abundance of Bacteroides, Faecalibacterium, Prevotella, and Lachnoclostridium genera was lower in patients with HT, and Blautia, Ruminococcus, Roseburia, Fusicatenibacter, Romboutsia, Dorea, and Eubacterium genera were higher in samples derived from patients with HT. Furthermore, the authors reported positive correlations between Firmicutes members and antiTPO or anti-Tg autoantibodies, while Bacteroidetes members inversely correlated with these antibodies. The abundance of Alloprevotella positively correlated with FT4 levels, while Romboutsia negatively correlated with TSH concentrations (24).

In a more recent cross-sectional work, Liu et al. (33) investigated the gut microbiota in 45 patients with euthyroid, 

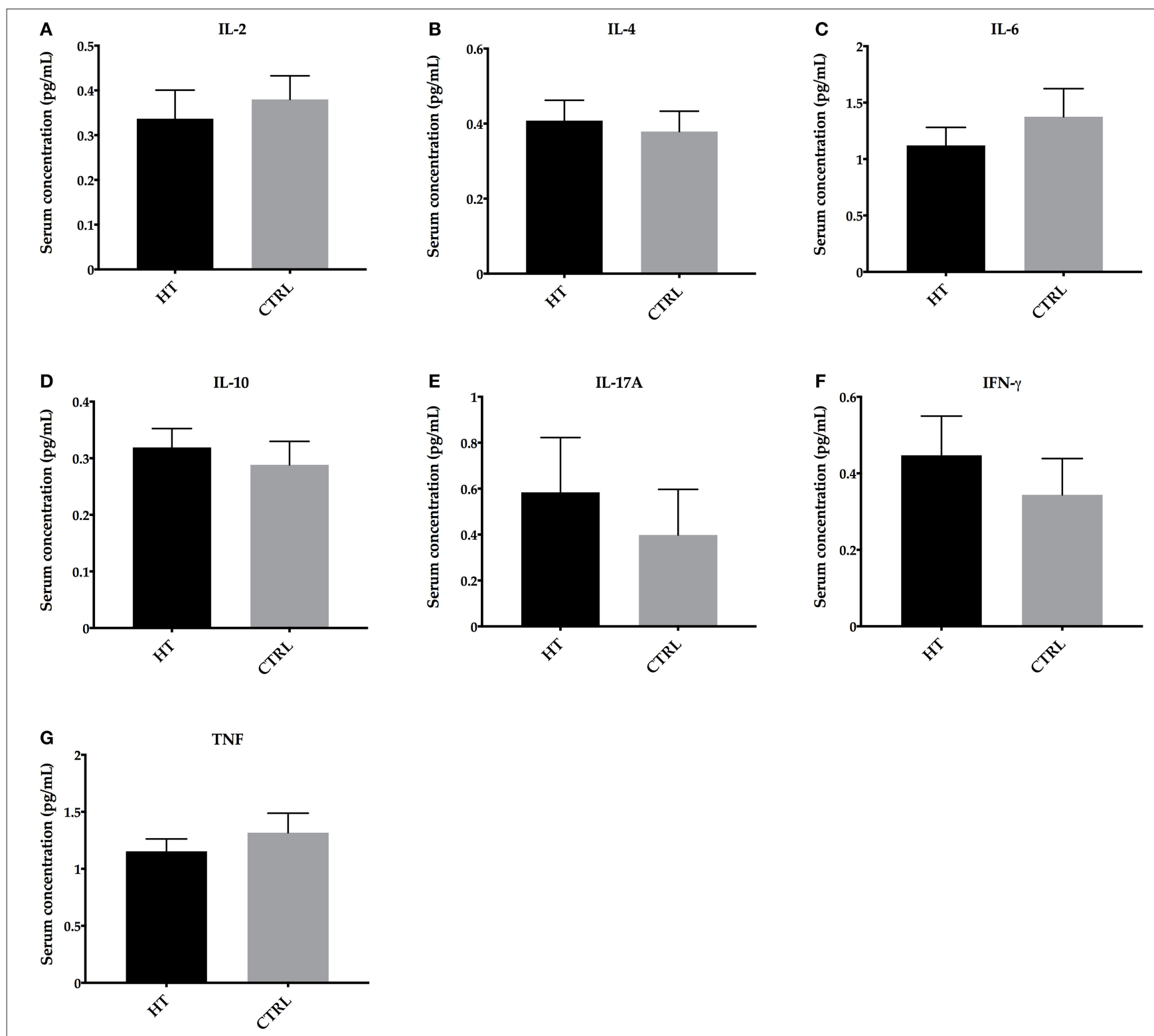

FIGURE 3 | Cytokine profile in HT patients and control subjects (CTRL). Serum concentrations of (A) IL-2, (B) IL-4, (C) IL-6, (D) IL-10, (E) IL-17A, (F) IFN- $\gamma$, and (G) TNF. Statistical analyses were performed by Mann-Whitney. Significance was set at $P<0.05$.

18 patients with HT hypothyroid, and 34 controls by $16 \mathrm{~S}$ sequencing. Compared with healthy controls, intestinal microbiota richness and diversity were significantly decreased in subjects with HT, with predominance of Bacteroidetes phylum members, Prevotella and Phascolarctobacterium species. In patients with euthyroid, some microbes, such as Lachnospiraceae incertae sedis, Lactonifactor, Alistipes, and Subdoligranulum genus, were enriched in stool samples. The authors concluded that there is a different microbiota profile in patients with diverse thyroid function, and they suggest that Phascolarctobacterium genus could be involved in HT progression in humans (33). Phascolarctobacterium species, mainly Phascolarctobacterium faecium, can produce acetate and propionate and influence the metabolic status of the host (35).

In accordance with the previous study, significant differences in richness and diversity of the gut microbiota were observed in 52 patients with primary hypothyroidism, compared with 40 healthy controls. The abundance of Bacteroidetes phylum members, Veillonella and Paraprevotella were significantly decreased, while Neisseria and Rheinheimera were increased in patients with hypothyroid. Veillonella and Paraprevotella genera positively correlated with FT3 and FT4 levels and were inversely associated with TSH concentrations. Researchers also showed that mRNA from enzymes involved in SCFAs production was decreased in the gut, and LPS serum levels were increased, 

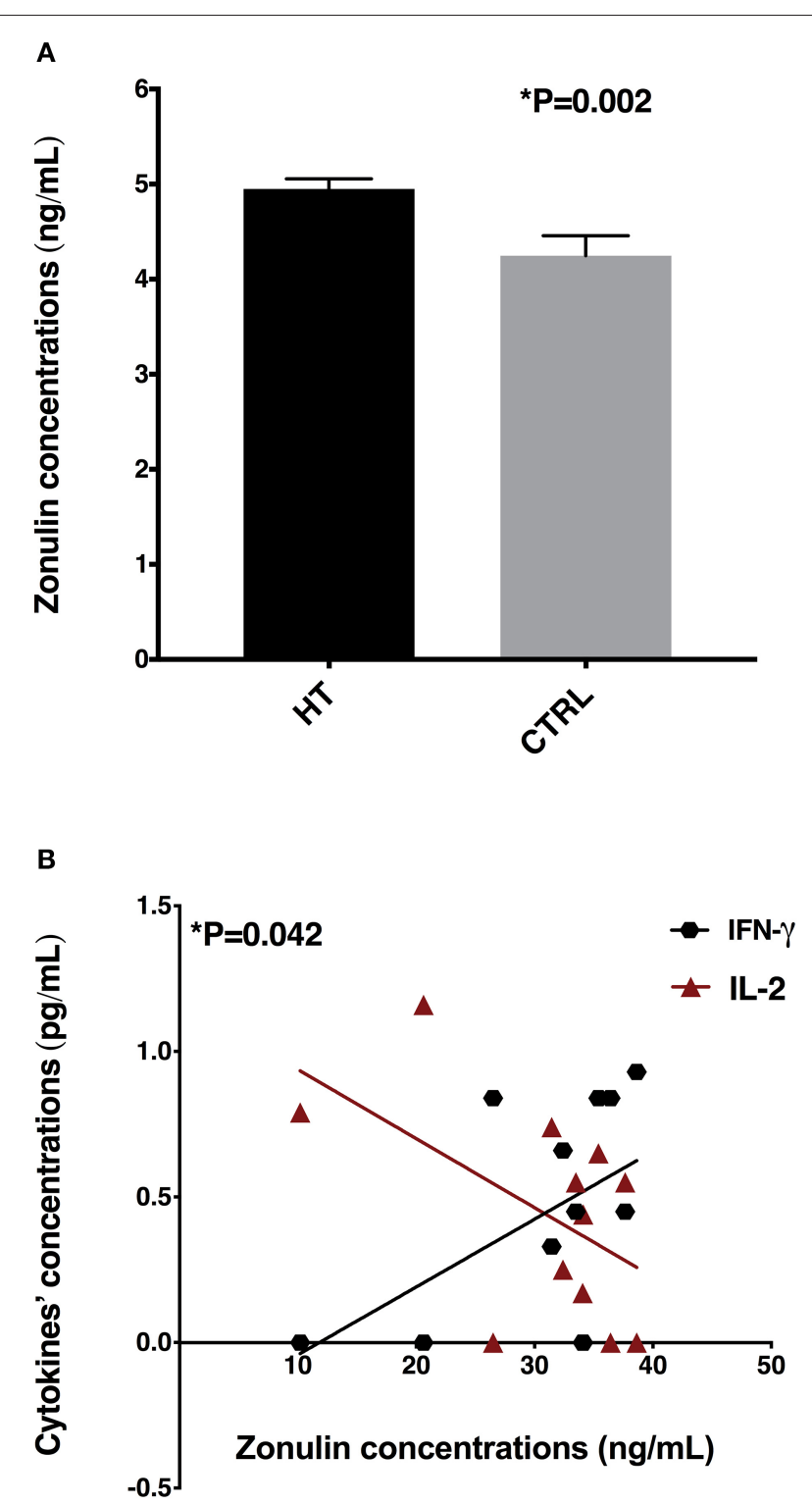

FIGURE 4 | Zonulin concentrations and correlations with systemic cytokine levels. (A) Serum zonulin concentrations in HT patients and controls (CTRL), (B) Correlations among zonulin levels with IFN- $\gamma$ and IL-2 concentrations.

suggesting a leaky gut in these patients. Additionally, mice that received fecal microbiota transplantation from patients with hypothyroid presented lower serum total thyroxine levels, decreased tight junction mRNA in the colon, and increased serum LPS (34).

There are neither any studies evaluating the gut microbiota in Brazilian patients with HT, nor are these data correlating with dietary habits, clinical data, and cytokines and zonulin concentrations. Here, we evaluated the gut microbiota in 40 Brazilian patients with HT and 53 healthy controls, and we observed a HT-associated microbiota imbalance, with an increase in Bacteroides and a significant decrease in
Bifidobacterium genus. Our data concerning the decreased abundance of Bifidobacterium are in agreement with real-time PCR results from Ishaq et al. (23). Bifidobacterium is the first microbial colonizer of the intestines in newborns, and it plays key roles in immune system maturation and dietary metabolism (36). Some Bifidobacterium strains are considered probiotic because of their beneficial effects, and some studies demonstrated the beneficial role of Bifidobacterium species in inflammatory conditions $(37,38)$. The discrepancies in relation to other studies and between studies may be due to different methodologies or even due to geographic location, lifestyle, and dietary habits.

In our study, we also observed significant differences in Lactobacillus abundance between patients who realized thyroid hormone replacement therapy and those who did not. Lactobacillus are acid-lactic producing bacteria and belong to the Firmicutes phylum, the second most prevalent in the human gut (39). Recently, a study showed that Lactobacillus promote gut barrier integrity by producing L-Ornithine from arginine amino acid (40). We suggest that this increase in Lactobacillus in patients with no hormone replacement could be associated with the small intestinal bacterial overgrowth (SIBO) in patients with HT (41). Lauritano et al. studied 50 patients with HT and 40 controls and found that $54 \%$ of patients presented SIBO, in contrast with the control group (4). A clinical trial conducted by Yao et al. (42) demonstrated that the intestinal microbiota in patients with hypothyroidism, randomly divided to receive L-thyroxine $(N=49)$ or no treatment $(N=$ 68), did not show significant differences with respect to alpha diversity. However, the relative abundance of Enterococcus and Odoribacter genera varied slightly depending on the dosage of L-thyroxine (42).

There are no studies correlating dietary components and gut microbiota in patients with HT. In our study, we found significant differences in dietary habits between patients with HT and controls regarding the frequency of the consumption of vegetables, fruits, animal-derived proteins, dairy products, saturated fats, and carbohydrates. Furthermore, we found an inverse correlation between animal-derived protein consumption and the abundance of Bacteroides genus. The main role of diet in microbiota modulation and its role in the host metabolism regulation can be exemplified by the establishment of the resident microbiota in early childhood, through the oligosaccharides ingestion from the breast milk, and also by the increase in the microbiota diversity associated with the solid food introduction $(43,44)$. The gut bacteria overgrowth can be directly affected by the nutrients, and these, in turn, affect both relative and absolute microbiota abundances and bacterial kinetics (45). Alterations in the ingestion of macronutrients, including proteins, carbohydrates, and fats, induce a significant shift in the gut microbiota (46). The microbiota modulation by diet directly influences on the inflammatory profile due to the generated microbiota metabolites and their direct or indirect action on immune cells from the mucosal immune system (4751). From fermentation of dietary fibers, the intestinal microbes can generate metabolites or SCFAs with anti-inflammatory properties and preserve gut homeostasis (47). In a recent study, 
Sepahi et al. showed that SCFAs produced by gut microbes in response to dietary fibers influence the expansion on innate lymphoid cells in the gut through G-protein-coupled receptors and can impact the immune responses in the distant tissues depending on the host condition (51). On the other hand, dietary fats are involved in decreased microbiota diversity and richness, which increased intestinal permeability and low-grade systemic inflammation (48).

In the present study, although we demonstrated significant differences in the dietary habits and alterations in the gut microbes in patients with HT, we did not observe significant differences in the cytokine profile in patients when compared with controls. We suggest that this factor can be related to patients' diet, lifestyle, or hormone replacement and disease control. In a recent study, Tabasi et al. (52) evaluated the gut microbiota by real-time PCR and cytokine concentrations in 23 patients with obese hypothyroid and 79 obese control subjects. Researchers reported no significant differences in the gut microbes between patients with hypothyroid and controls. However, similar to our findings, the serum cytokine concentrations, including IL$1 \beta$, IL-6, IFN- $\gamma$, and TGF- $\beta$, were similar in patients and controls (52).

Finally, although there are no differences in systemic cytokines in patients with $\mathrm{HT}$, we detected an increased intestinal permeability, seen by the higher zonulin concentrations in the serum of patients, when compared with controls. Similar to our study, a case-control work investigated the leaky gut in 30 patients with HT (children and adolescents), and 30 patients with congenital hypothyroidism, and reported a significant increase in serum zonulin in patients with HT. Moreover, zonulin levels positively correlated with levothyroxine dose, suggesting a connection between the increased intestinal permeability and disease severity (53). Zonulin is a physiological modulator of intercellular tight junctions, involved in macromolecules trafficking, epithelial and endothelial barrier integrity, and immune tolerance in the gut mucosa (54). Intestinal dysbiosis can activate the zonulin pathway and stimulate their release and allow the traffic of luminal contents through the epithelial barrier. The leaky gut induces inflammatory cytokines release that themselves promote an increased permeability, a vicious circle favoring the entry of antigens derived from diet and gut microbes, triggering the activation of innate and adaptive immunity in the gut mucosa (55). The main factors involved in zonulin release are the bacterial overgrowth and gluten, and the increased intestinal permeability can induce a tolerance breakdown, and then, activated immune cells can remain in the gut mucosa or migrate to distant organs, participating in chronic inflammatory and autoimmune diseases (55-57).

\section{REFERENCES}

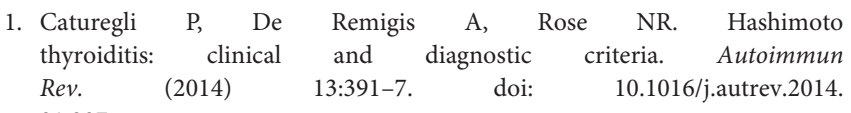

\section{CONCLUSIONS}

We concluded that there are alterations in the microbiota and intestinal permeability in Brazilian patients with HT. In addition, we suggest that diet might have played an important role in modulating the gut microbiota in patients with HT. These findings could help understand the HT development and progression, while further investigations to clarify the underlying mechanisms of the diet-microbiota-immune system axis are still needed.

\section{DATA AVAILABILITY STATEMENT}

The original contributions presented in the study are included in the article/supplementary material, further inquiries can be directed to the corresponding author/s.

\section{ETHICS STATEMENT}

The studies involving human participants were reviewed and approved by Barretos Cancer Hospital Ethics Committee (Process number 1,359/2017). The patients/participants provided their written informed consent to participate in this study.

\section{AUTHOR CONTRIBUTIONS}

LC and LVVS: enrollment of patients with HT, DNA extraction and quantification, cytokine determination, data acquisition, and manuscript writing. LC, GR, and AB: enrollment of controls and sample collection. AL: PCR real-time experiments and cytokine determination. LDBS: responsible for clinical data from patients with HT. JB: support for blood samples collection. EG: manuscript writing and revision. GO: experimental design, data interpretation, manuscript writing, and revision. All authors contributed to the article and approved the submitted version.

\section{FUNDING}

This study was supported by the Brazilian Governmental Agency, Fundação de Amparo à Pesquisa do Estado de São Paulo (FAPESP Grant numbers \#2017/07444-2 and 2021/01035-9), and by the School of Health Sciences from Barretos Dr. Paulo Prata (PAP Grant number \#2017).

\section{ACKNOWLEDGMENTS}

We would like to thank the Microbiology Program from Institute of Biosciences, Humanities, and Exact Sciences (IBILCE), São Paulo State University (UNESP), Sao Jose do Rio Preto, Brazil.

2. Fröhlich E, Wahl R. Thyroid autoimmunity: role of anti-thyroid antibodies in thyroid and extra-thyroidal diseases. Front Immunol. (2017) 8:521. doi: 10.3389/fimmu.2017.00521

3. Ajjan RA, Weetman AP. The pathogenesis of Hashimoto's thyroiditis: further developments in our understanding. Horm Metab Res. (2015) 47:70210. doi: $10.1055 / \mathrm{s}-0035-1548832$ 
4. Lauritano EC, Bilotta AL, Gabrielli M, Scarpellini E, Lupascu A, Laginestra A, et al. Association between hypothyroidism and small intestinal bacterial overgrowth. J Clin Endocrinol Metab. (2007) 92:4180-4. doi: 10.1210/jc.2007-0606

5. Chiovato L, Magri F, Carlé A. Hypothyroidism in context: where we've been and where we're going. Adv Ther. (2019) 36(Suppl. 2):4758. doi: 10.1007/s12325-019-01080-8

6. Chaker L, Bianco AC, Jonklaas J, Peeters RP. Hypothyroidism. Lancet Lond Engl. (2017) 390:1550-62. doi: 10.1016/S0140-6736(17)30703-1

7. Hiromatsu Y, Satoh H, Amino N. Hashimoto's thyroiditis: history and future outlook. Horm Athens Greece. (2013) 12:12-8. doi: 10.1007/BF03 401282

8. Garmendia Madariaga A, Santos Palacios S, Guillén-Grima F, Galofré JC. The incidence and prevalence of thyroid dysfunction in Europe: a meta-analysis. $J$ Clin Endocrinol Metab. (2014) 99:923-31. doi: 10.1210/jc.2013-2409

9. Sgarbi JA, Teixeira PFS, Maciel LMZ, Mazeto GMFS, Vaisman M, Montenegro Junior RM, et al. The Brazilian consensus for the clinical approach and treatment of subclinical hypothyroidism in adults: recommendations of the thyroid Department of the Brazilian Society of Endocrinology and Metabolism. Arq Bras Endocrinol Amp Metabol. (2013) 57:16683. doi: 10.1590/S0004-27302013000300003

10. Loh HH, Lim LL, Yee A, Loh HS. Association between subclinical hypothyroidism and depression: an updated systematic review and metaanalysis. BMC Psychiatry. (2019) 19:12. doi: 10.1186/s12888-018-2006-2

11. Qin J, Yu Z, Guan H, Shi L, Liu Y, Zhao N, et al. High thyroglobulin antibody levels increase the risk of differentiated thyroid carcinoma. Dis Markers. (2015) 2015:648670. doi: 10.1155/2015/648670

12. Jankovic B, Le KT, Hershman JM. Clinical review: Hashimoto's thyroiditis and papillary thyroid carcinoma: is there a correlation? J Clin Endocrinol Metab. (2013) 98:474-82. doi: 10.1210/jc.2012-2978

13. Prinzi N, Sorrenti S, Baldini E, De Vito C, Tuccilli C, Catania A, et al. Association of thyroid diseases with primary extra-thyroidal malignancies in women: results of a cross-sectional study of 6,386 patients. PLoS ONE. (2015) 10:e0122958. doi: 10.1371/journal.pone.0122958

14. Taylor PN, Albrecht D, Scholz A, Gutierrez-Buey G, Lazarus JH, Dayan CM, et al. Global epidemiology of hyperthyroidism and hypothyroidism. Nat Rev Endocrinol. (2018) 14:301-16. doi: 10.1038/nrendo.2018.18

15. Liontiris MI, Mazokopakis EE. A concise review of Hashimoto thyroiditis (HT) and the importance of iodine, selenium, vitamin D and gluten on the autoimmunity and dietary management of HT patients. Points that need more investigation. Hell J Nucl Med. (2017) 20:51-6. doi: 10.1967/s002449910507

16. Köhling HL, Plummer SF, Marchesi JR, Davidge KS, Ludgate M. The microbiota and autoimmunity: their role in thyroid autoimmune diseases. Clin Immunol. (2017) 183:63-74. doi: 10.1016/j.clim.2017.07.001

17. Fröhlich E, Wahl R. Microbiota and thyroid interaction in health and disease. Trends Endocrinol Metab TEM. (2019) 30:479-90. doi: 10.1016/j.tem.2019.05.008

18. Knezevic J, Starchl C, Tmava Berisha A, Amrein K. Thyroid-gut-axis: how does the microbiota influence thyroid function? Nutrients. (2020) 12:1769. doi: $10.3390 /$ nu12061769

19. Levy M, Kolodziejczyk AA, Thaiss CA, Elinav E. Dysbiosis and the immune system. Nat Rev Immunol. (2017) 17:219-32. doi: 10.1038/nri.2017.7

20. Meng S, Badrinarain J, Sibley E, Fang R, Hodin R. Thyroid hormone and the d-type cyclins interact in regulating enterocyte gene transcription. J Gastrointest Surg Off J Soc Surg Aliment Tract. (2001) 5:49-55. doi: 10.1016/S1091-255X(01)80013-5

21. Song Y, Zhao M, Zhang H, Zhang X, Zhao J, Xu J, et al. Thyroid-stimulating hormone levels are inversely associated with serum total bile acid levels: a cross-sectional study. Endocr Pract Off J Am Coll Endocrinol Am Assoc Clin Endocrinol. (2016) 22:420-6. doi: 10.4158/EP15844.OR

22. Fenneman AC, Rampanelli E, Yin YS, Ames J, Blaser MJ, Fliers E, et al. Gut microbiota and metabolites in the pathogenesis of endocrine disease. Biochem Soc Trans. (2020) 48:915-31. doi: 10.1042/BST20190686

23. Ishaq HM, Mohammad IS, Guo H, Shahzad M, Hou YJ, Ma C, et al. Molecular estimation of alteration in intestinal microbial composition in Hashimoto's thyroiditis patients. Biomed Pharmacother Biomedecine Pharmacother. (2017) 95:865-74. doi: 10.1016/j.biopha.2017.08.101
24. Zhao F, Feng J, Li J, Zhao L, Liu Y, Chen H, et al. Alterations of the gut microbiota in Hashimoto's thyroiditis patients. Thyroid Off J Am Thyroid Assoc. (2018) 28:175-86. doi: 10.1089/thy.2017.0395

25. Larsen N, Vogensen FK, van den Berg FWJ, Nielsen DS, Andreasen AS, Pedersen BK, et al. Gut microbiota in human adults with type 2 diabetes differs from non-diabetic adults. PLoS ONE. (2010) 5:e9085. doi: 10.1371/journal.pone.0009085

26. Rodrigues GSP, Cayres LCF, Gonçalves FP, Takaoka NNC, Lengert AH, Tansini A, et al. Detection of increased relative expression units of Bacteroides and Prevotella, and decreased Clostridium leptum in stool samples from Brazilian rheumatoid arthritis patients: a pilot study. Microorganisms. (2019) 7:413. doi: 10.3390/microorganisms7100413

27. Lerner A, Aminov R, Matthias T. Dysbiosis may trigger autoimmune diseases via inappropriate post-translational modification of host proteins. Front Microbiol. (2016) 7:84. doi: 10.3389/fmicb.2016.00084

28. de Oliveira GLV, Leite AZ, Higuchi BS, Gonzaga MI, Mariano VS. Intestinal dysbiosis and probiotic applications in autoimmune diseases. Immunology. (2017) 152:1-12. doi: 10.1111/imm.12765

29. De Luca F, Shoenfeld Y. The microbiome in autoimmune diseases. Clin Exp Immunol. (2019) 195:74-85. doi: 10.1111/cei.13158

30. Lerner A, Jeremias P, Matthias T. Gut-thyroid axis and celiac disease. Endocr Connect. (2017) 6:R52-8. doi: 10.1530/EC-17-0021

31. $\mathrm{Mu}$ Q, Kirby J, Reilly CM, Luo XM. Leaky gut as a danger signal for autoimmune diseases. Front Immunol. (2017) 8:598. doi: 10.3389/fimmu.2017.00598

32. Rooks MG, Garrett WS. Gut microbiota, metabolites and host immunity. Nat Rev Immunol. (2016) 16:341-52. doi: 10.1038/nri.2016.42

33. Liu S, An Y, Cao B, Sun R, Ke J, Zhao D. The composition of gut microbiota in patients bearing Hashimoto's thyroiditis with euthyroidism and hypothyroidism. Int $J$ Endocrinol. (2020) 2020:5036959. doi: 10.1155/2020/5036959

34. Su X, Zhao Y, Li Y, Ma S, Wang Z. Gut dysbiosis is associated with primary hypothyroidism with interaction on gut-thyroid axis. Clin Sci Lond Engl. (1979) (2020) 134:1521-35. doi: 10.1042/CS20200475

35. Wu F, Guo X, Zhang J, Zhang M, Ou Z, Peng Y. Phascolarctobacterium faecium abundant colonization in human gastrointestinal tract. $\operatorname{Exp}$ Ther Med. (2017) 14:3122-6. doi: 10.3892/etm.2017.4878

36. O'Callaghan A, van Sinderen D. Bifidobacteria and their role as members of the human gut microbiota. Front Microbiol. (2016) 7:925. doi: $10.3389 /$ fmicb. 2016.00925

37. Patole SK, Rao SC, Keil AD, Nathan EA, Doherty DA, Simmer $\mathrm{KN}$. Benefits of Bifidobacterium breve $\mathrm{M}-16 \mathrm{~V}$ supplementation in preterm neonates - a retrospective cohort study. PLoS ONE. (2016) 11:e0150775. doi: 10.1371/journal.pone.0150775

38. Saez-Lara MJ, Gomez-Llorente C, Plaza-Diaz J, Gil A. The role of probiotic lactic acid bacteria and bifidobacteria in the prevention and treatment of inflammatory bowel disease and other related diseases: a systematic review of randomized human clinical trials. BioMed Res Int. (2015) 2015:505878. doi: 10.1155/2015/505878

39. Azad MAK, Sarker M, Li T, Yin J. Probiotic species in the modulation of gut microbiota: an overview. BioMed Res Int. (2018) 2018:9478630. doi: 10.1155/2018/9478630

40. Qi H, Li Y, Yun H, Zhang T, Huang Y, Zhou J, et al. Lactobacillus maintains healthy gut mucosa by producing L-Ornithine. Commun Biol. (2019) 2:171. doi: 10.1038/s42003-019-0424-4

41. Patil AD. Link between hypothyroidism and small intestinal bacterial overgrowth. Indian J Endocrinol Metab. (2014) 18:3079. doi: 10.4103/2230-8210.131155

42. Yao Z, Zhao M, Gong Y, Chen W, Wang Q, Fu Y, et al. Relation of gut microbes and L-thyroxine through altered thyroxine metabolism in subclinical hypothyroidism subjects. Front Cell Infect Microbiol. (2020) 10:495. doi: 10.3389/fcimb.2020.00495

43. Laursen MF, Bahl MI, Michaelsen KF, Licht TR. First foods and gut microbes. Front Microbiol. (2017) 8:356. doi: 10.3389/fmicb.2017.00356

44. Zmora N, Suez J, Elinav E. You are what you eat: diet, health and the gut microbiota. Nat Rev Gastroenterol Hepatol. (2019) 16:3556. doi: 10.1038/s41575-018-0061-2 
45. Korem T, Zeevi D, Suez J, Weinberger A, Avnit-Sagi T, Pompan-Lotan $\mathrm{M}$, et al. Growth dynamics of gut microbiota in health and disease inferred from single metagenomic samples. Science. (2015) 349:11016. doi: $10.1126 /$ science.aac4812

46. Kolodziejczyk AA, Zheng D, Elinav E. Diet-microbiota interactions and personalized nutrition. Nat Rev Microbiol. (2019) 17:74253. doi: 10.1038/s41579-019-0256-8

47. Yap YA, Mariño E. An insight into the intestinal web of mucosal immunity, microbiota, and diet in inflammation. Front Immunol. (2018) 9:2617. doi: 10.3389/fimmu.2018.02617

48. Cândido FG, Valente FX, Grześkowiak ŁM, Moreira APB, Rocha DMUP, Alfenas R de CG. Impact of dietary fat on gut microbiota and low-grade systemic inflammation: mechanisms and clinical implications on obesity. Int J Food Sci Nutr. (2018) 69:125-43. doi: 10.1080/09637486.2017.1343286

49. Zou J, Chassaing B, Singh V, Pellizzon M, Ricci M, Fythe MD, et al. Fibermediated nourishment of gut microbiota protects against diet-induced obesity by restoring IL-22-mediated colonic health. Cell Host Microbe. (2018) 23:4153.e4. doi: 10.1016/j.chom.2017.11.003

50. Matt SM, Allen JM, Lawson MA, Mailing LJ, Woods JA, Johnson RW. Butyrate and dietary soluble fiber improve neuroinflammation associated with aging in mice. Front Immunol. (2018) 9:1832. doi: 10.3389/fimmu.2018.01832

51. Sepahi A, Liu Q, Friesen L, Kim CH. Dietary fiber metabolites regulate innate lymphoid cell responses. Mucosal Immunol. (2020) 15:1-14. doi: 10.1038/s41385-020-0312-8

52. Tabasi M, Eybpoosh S, Sadeghpour Heravi F, Siadat SD, Mousavian G, Elyasinia F, et al. Gut microbiota and serum biomarker analyses in obese patients diagnosed with diabetes and hypothyroid disorder. Metab Syndr Relat Disord. (2020) 24:1-8. doi: 10.21203/rs.3.rs-46107/v1

53. Küçükemre Aydin B, Yildiz M, Akgün A, Topal N, Adal E, Önal H. Children with Hashimoto's thyroiditis have increased intestinal permeability: results of a pilot study. J Clin Res Pediatr Endocrinol. (2020) 12:3037. doi: 10.4274/jcrpe.galenos.2020.2019.0186

54. Fasano A. Zonulin and its regulation of intestinal barrier function: the biological door to inflammation, autoimmunity, and cancer. Physiol Rev. (2011) 91:151-75. doi: 10.1152/physrev.00003.2008

55. Fasano A. All disease begins in the (leaky) gut: role of zonulin-mediated gut permeability in the pathogenesis of some chronic inflammatory diseases. F1000Research. (2020) 9:1-12. doi: 10.12688/f1000research. 20510.1

56. Drago S, El Asmar R, Di Pierro M, Grazia Clemente M, Tripathi A, Sapone A, et al. Gliadin, zonulin and gut permeability: effects on celiac and non-celiac intestinal mucosa and intestinal cell lines. Scand J Gastroenterol. (2006) 41:408-19. doi: 10.1080/003655205002 35334

57. El Asmar R, Panigrahi P, Bamford P, Berti I, Not T, Coppa GV, et al. Hostdependent zonulin secretion causes the impairment of the small intestine barrier function after bacterial exposure. Gastroenterology. (2002) 123:160715. doi: 10.1053 /gast.2002.36578

Conflict of Interest: The authors declare that the research was conducted in the absence of any commercial or financial relationships that could be construed as a potential conflict of interest.

Copyright (C) 2021 Cayres, de Salis, Rodrigues, Lengert, Biondi, Sargentini, Brisotti, Gomes and de Oliveira. This is an open-access article distributed under the terms of the Creative Commons Attribution License (CC BY). The use, distribution or reproduction in other forums is permitted, provided the original author(s) and the copyright owner(s) are credited and that the original publication in this journal is cited, in accordance with accepted academic practice. No use, distribution or reproduction is permitted which does not comply with these terms. 